\title{
Photochemical Synthesis of Ag and Au Nanoparticles Using a Thioxanthone Substituted Chitosan as Simultaneous Photoinitiator and Stabilizer
}

\author{
Silvano R. Valandro, ${ }^{a}$ Alessandra L. Poli, ${ }^{a}$ Miguel G. Neumann ${ }^{a}$ and Carla C. Schmitt ${ }^{\circledR *, a}$ \\ ${ }^{a}$ Instituto de Química de São Carlos, Universidade de São Paulo, \\ CP 780, 13560-970 São Carlos-SP, Brazil
}

\begin{abstract}
Silver (AgNPs) and gold (AuNPs) nanoparticles in aqueous solution were generated and stabilized using the 10-oxo-10H-dibenzene thiopyran-3-4-dicarboximide chitosan (TXICh) macrophotoinitiator. The photoinitiator and the nanoparticles in aqueous solution were characterized by UV-Vis spectroscopy and transmission electron microscopy (TEM). Pure TXICh in aqueous solution forms self-assembled particles with diameter around $30 \mathrm{~nm}$. AgNPs were prepared using TXICh in the presence and absence of triethanolamine (TEOH). During irradiation, a broad band in the range of 410-415 $\mathrm{nm}$ was observed, corresponding to the plasmon absorption, indicating the AgNPs formation. TEM images revealed that the AgNPs had spherical shape in the absence and presence of TEOH. The synthesis of AgNPs in the presence of TEOH occurred rapidly (maximum conversion reached after $15 \mathrm{~min}$ ), whereas in its absence 4 hours were required. The AgNPs prepared with TXICh/TEOH presented smaller and more uniform nanoparticles size. AuNPs were also prepared using TXICh as photoinitiator/stabilizer system. These nanoparticles showed an intense surface plasmon band at $530 \mathrm{~nm}$ and the TEM images revealed spherical particles of around $4 \mathrm{~nm}$. These studies indicate that TXICh can act not only as a photoinitiator, but also as a stabilizer in the AgNPs and AuNPs formation.
\end{abstract}

Keywords: silver nanoparticles, gold nanoparticles, nanoparticle photochemical synthesis, chitosan macrophotoinitiator, simultaneous photoinitiator and stabilizer

\section{Introduction}

In recent years silver and gold nanostructures have found countless application in a wide range of areas due to their antimicrobial,,$^{1,2}$ optical,,$^{3,4}$ electronic $^{5,6}$ and catalytic ${ }^{7,8}$ properties. Several methods have been reported for the preparation of silver (AgNPs) and gold (AuNPs) nanoparticles, including chemical reduction, ${ }^{9,10}$ UV irradiation ${ }^{11}$ and pulsed laser ablation. ${ }^{12-14}$ The photochemical synthesis of nanoparticles presents advantages over other methods due to the absence of other reductive chemicals in the system that might contaminate the nanoparticles and affect its properties when used in practical applications. Photochemical syntheses of metal nanoparticles can be performed by direct photoreduction of the corresponding metal complex, or by photosensitization using an appropriate photosensitizer. ${ }^{15}$

AgNPs and AuNPs can be stabilized by clays, ${ }^{16,17}$ low molecular weight ligands ${ }^{18-20}$ polymers and/or biopolymers. ${ }^{21,22}$ In systems where the nanoparticles are

*e-mail: carla@iqsc.usp.br assembled into these materials, the stabilizing agent is used as a matrix to promote the homogeneity, prevent the agglomeration and precipitation of the metal nanoparticles, ensuring long-term stability.

Chitosan, which is an aminopolysaccharide composed of $\beta$ - $(1 \rightarrow 4)$-linked $N$-acetyl-D-glucosamine and D-glucosamine units, ${ }^{23-26}$ has been used as a stabilizer of gold and silver nanoparticles by different preparation methods. ${ }^{27-32}$ It has been reported that chitosan can be used as a stabilizer as well as reducing reagent by UV irradiation in the preparation of silver nanoparticles. ${ }^{33}$ However, the reaction could only be performed in acidic media due to the poor solubility of chitosan in neutral and basic media.

We recently developed an efficient chitosan-based photoinitiator by functionalizing chitosan with 10-oxo10H-dibenzene thiopyran-3-4-dicarboximide (TXI), a thioxanthone derivative (Figure 1) ${ }^{34}$ It was shown that the chitosan chains acted as a support for anchoring the TXI units, resulting in a new macromolecular photopolymerization initiator that kept the main photochemical properties of the small thioxanthone, such as wide absorption spectrum 
and high rate of intersystem crossing and triplet quantum yield formation. ${ }^{35-40}$

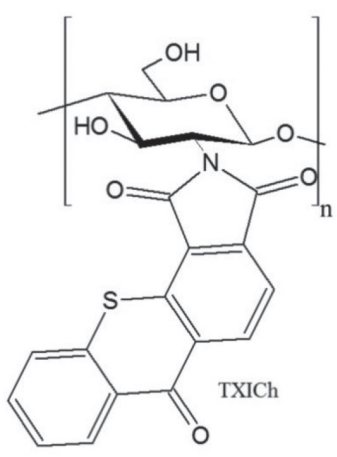

Figure 1. Structure of thioxanthone derivative bound to chitosan (TXICh).

We want to report here a new photochemical synthesis of silver and gold nanoparticles by using a macrophotoinitiator consisting of thioxanthone bound to chitosan (TXICh). This amphiphilic macrophotoinitiator can act as a reducing agent promoting the photoredution of the metallic ions, as well as a stabilizer of the formed metal nanoparticles. The obtained nanoparticles were characterized by UV-Vis spectroscopy and transmission electron microscopy.

\section{Experimental}

Materials

Silver nitrate $\left(\mathrm{AgNO}_{3}\right.$, TEC-LAB, São Paulo, Brazil), gold(III) chloride trihydrate $\left(\mathrm{HAuCl}_{4} \cdot 3 \mathrm{H}_{2} \mathrm{O}\right.$; Sigma Aldrich, USA) and triethanolamine (TEOH; Synt, Diadema, Brazil) were used as received.

Functionalization of chitosan with $10-0 x 0-10 \mathrm{H}$-dibenzene thiopyran-3-4-dicarboximide

The 10-oxo-10H-dibenzene thiopyran3-4-dicarboximide/chitosan (TXICh) was synthesized by phthaloylation of chitosan, followed by reaction with thiosalicyclic acid in a mixture of methanesulfonic acid and $\mathrm{Al}_{2} \mathrm{O}_{3}$, as describe previously. ${ }^{34}$

\section{Synthesis Ag/TXICh and Au/TXICh nanoparticles}

Silver nanoparticles were prepared in aqueous solution of TXICh $\left(0.025 \mathrm{~g} \mathrm{~L}^{-1}\right), \mathrm{AgNO}_{3}\left(2 \times 10^{-4} \mathrm{~mol} \mathrm{~L}^{-1}\right)$ in the absence and presence of triethanolamine $\left(1.5 \times 10^{-2} \mathrm{~mol} \mathrm{~L}^{-1}\right)$. The solutions were irradiated with a $365-n m$ UV-LED (92 $\mathrm{mW} \mathrm{cm}^{-2}$, THORLAB M365L2).

Gold nanoparticles were prepared from an aqueous solution of $\mathrm{HAuCl}_{4}\left(3.3 \times 10^{-4} \mathrm{~mol} \mathrm{~L}^{-1}\right)$ in the presence of TXICh $\left(0.025 \mathrm{~g} \mathrm{~L}^{-1}\right)$ and TEOH $\left(5 \times 10^{-3} \mathrm{~mol} \mathrm{~L}^{-1}\right)$. The samples were irradiated using a 405-nm blue light emitting

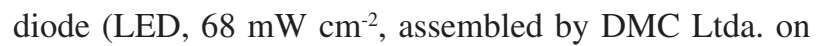
demand). The AgNPs photosyntheses were performed using a 365-nm LED to avoid the irradiation of the plasmon band at ca. $410 \mathrm{~nm}$ during the process.

All the samples were placed in a $3 \mathrm{~mL}$ cuvette at room temperature (ca. $25^{\circ} \mathrm{C}$ ) and purged with nitrogen for $15 \mathrm{~min}$ before irradiation. Irradiations were performed for a few minutes after the stabilization of the plasmon band. The irradiance of the LEDs was measured using a spectroradiometer (LUZCHEM SPR-01). No further treatment was performed after the end of the irradiation.

\section{Characterization}

The formation of AgNPs was monitored by UV-Vis absorption using a Shimadzu UV-2550 Spectrophotometer. Fluorescence measurements were performed on a Hitachi F-4500 spectrofluorometer at room temperature with excitation at $390 \mathrm{~nm}$.

Dynamic light scattering (DLS) analysis of TXICh was performed using a Zetasizer Nano ZS analyser. The morphological characterization of the TXICh was performed using a FEI-TECNAI G2 -F20 microscope. Samples were prepared by deposition of TXICh $0.025 \mathrm{~g} \mathrm{~L}^{-1}$ aqueous solution on carbon coated $\mathrm{Cu}$ minigrids $(\mathrm{CF}-200 \mathrm{Cu}$, Electron Microscopy Sciences). The minigrids were allowed to dry for $20 \mathrm{~min}$ and post-stained with phosphotungstenic acid at $2 \%$.

The morphology size distributions of the Ag and Au nanoparticles were obtained by transmission electron microscope (TEM) (JEOL JEM2100 LaB 6 -200 kV). The samples were drop-casted on carbon coated $\mathrm{Cu}$ minigrids.

\section{Results and Discussion}

\section{Characterization of TXICh in aqueous solution}

TXICh in aqueous solution shows an absorption over a wide region of the visible spectrum (Figure $2 \mathrm{a}$ ), which makes it a promising photoinitiator for the synthesis of metal nanoparticles. The absorption over the $350-430 \mathrm{~nm}$ range corresponds to the $\mathrm{n} \pi *$ and $\pi \pi^{*}$ electronic transitions. From the fluorescence emission spectrum (Figure 2a), it is possible to assign the band at $445 \mathrm{~nm}$ to the $S_{1}$ state. ${ }^{34}$

The TEM image (Figure $2 \mathrm{~b}$ and inset) suggests that in aqueous solution TXICh forms spherical self-assembled particles with diameters around $30 \mathrm{~nm}$. Similar results were observed from DLS measurements (Figure S2, Supplementary Information (SI) section). 

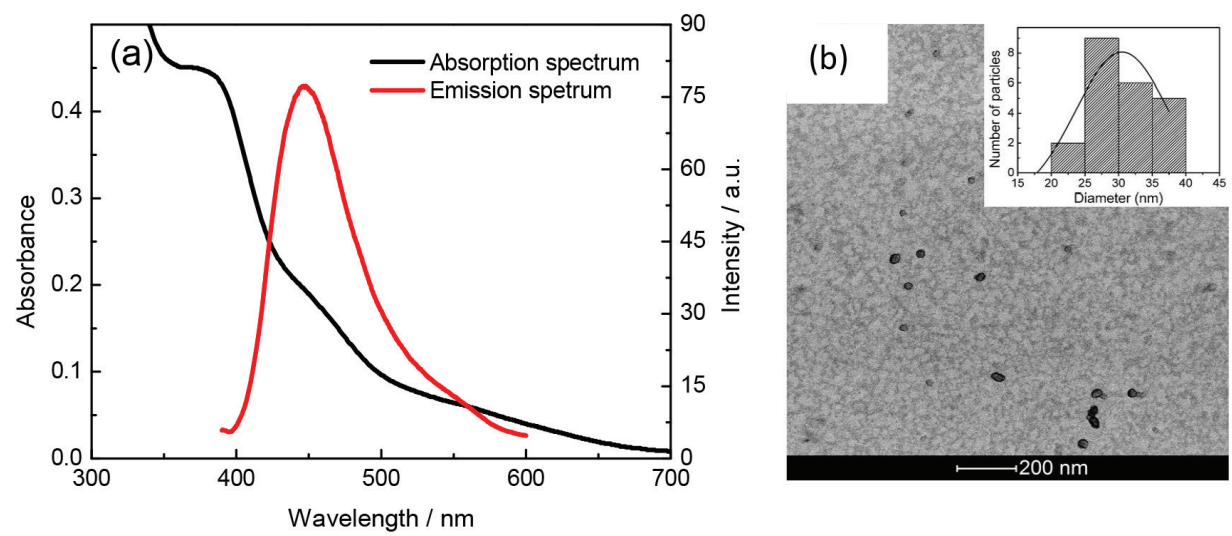

Figure 2. (a) UV-Vis absorption and fluorescence emission spectra of TXICh in aqueous solution; (b) transmission electron micrographs of TXICh post-stained with $2 \%$ phosphotungstenic acid. (A more detailed TEM image can be seen in Figure S1, SI section).

\section{Synthesis and characterization of AgNPs/TXICh}

Initially, TXICh was used as a photoreducing and stabilizer agent for the photochemical preparation of AgNPs. A solution of silver nitrate was irradiated with a $365-\mathrm{nm}$ UV-LED in the presence of TXICh. The formation of AgNPs was monitored by UV-Vis absorption (Figure 3a). It is worth noting that the appearance of plasmon band around $415 \mathrm{~nm}$ was observed only when the solution was irradiated.

The formation of AgNPs occurs when the thioxanthone groups attached to chitosan (TXICh) absorb light, being excited to its singlet excited state and undergoing intersystem crossing to the triplet excited state. ${ }^{34,41}$ The hydrogen transfer from the solvent and/or chitosan chains to the triplet state, results in the formation of a ketyl radical $[(\mathrm{H}) \mathrm{TXICh}]^{\cdot 42}$ This radical may induce the redox process to initiate the generation of the AgNPs. The other possible radicals $\mathrm{R}(-\mathrm{H})^{\circ}$ originated from the abstraction of hydrogen have lower reduction potential and will not interfere in the process.

$$
\begin{aligned}
& {[\mathrm{TXICh}]+\mathrm{h} \nu \rightarrow{ }^{1}[\mathrm{TXICh}]^{*}} \\
& { }^{1}[\mathrm{TXICh}]^{*} \rightarrow{ }^{3}[\mathrm{TXICh}]^{*} \\
& { }^{3}\left[\mathrm{TXICh}^{*}+\mathrm{R}(\mathrm{H}) \rightarrow[(\mathrm{H}) \mathrm{TXICh}]^{\bullet}+\mathrm{R}(-\mathrm{H})^{\bullet}\right.
\end{aligned}
$$

The formation of the nanoparticles due to the reaction of the $\mathrm{Ag}^{+}$ions with the chitosan derivative free radicals [(H)TXICh $]^{\bullet}$ means that the AgNPs are formed within the frame of the chitosan and, therefore, promptly stabilized.

The addition of a more effective radical source to the reaction system was explored by adding triethanolamine to the reacting solution. Triethanolamine is an aliphatic amine with a good hydrogen donor potential, so that it can be successfully used as an alternative radical source.

$$
{ }^{3}\left[\mathrm{TXICh}^{*}+\mathrm{TEOH} \rightarrow[(\mathrm{H}) \mathrm{TXICh}]^{\bullet}+[\mathrm{TEOH}(-\mathrm{H})]^{\bullet}\right.
$$

where $[\mathrm{TEOH}(-\mathrm{H})]^{\bullet}=\left(\mathrm{CH}_{2} \mathrm{OH}-\mathrm{CH}_{2}\right)_{2} \mathrm{NC} \cdot \mathrm{H}-\mathrm{CH}_{2} \mathrm{OH}$.
Additionally, some of the $[\mathrm{TEOH}(-\mathrm{H})]^{\bullet}$ radicals formed within the chitosan environment may regenerate the ground state dye before migrating out to reduce the metal ions, so that the light absorbing species will not be depleted.

Figures $3 \mathrm{a}$ and $3 \mathrm{~b}$ show the UV-Vis absorption spectra of AgNPs/TXICh/ and AgNPs/TXICh/TEOH as a function of irradiation time. The plasmon surface band for the amine-containing system shows a small blue shift $(410 \mathrm{~nm})$ compared to the system without amine. This band presents a narrow and symmetrical shape, which is an indicative of a narrow particle size distribution. ${ }^{43}$ Moreover, the maximum conversion of the silver salt into AgNPs was reached after about $15 \mathrm{~min}$ of irradiation of the $\mathrm{AgNO}_{3} / \mathrm{TXICh} / \mathrm{TEOH}$ system. This behavior can be attributed to the presence of amine radicals. In this case, the main hydrogen transfer occurs from the TEOH to the TXICh triplet exited state, resulting in the semireduced form of the ketone and amine radicals. ${ }^{34}$ These radicals proved to be extremely efficient for the photoreduction of $\mathrm{Ag}^{+}$ions.

The AgNPs morphology was evaluated by transmission electron microscopy. Nanoparticles prepared in the absence of TEOH (Figures 4a and 4b) showed multiple twinning and stacking faults. Besides, a non-uniform particle size was observed, resulting in a polydispersed particle size distribution in the 2-24 $\mathrm{nm}$ range (inset of Figure 4b). On the other hand, these characteristic were not observed for the $\mathrm{AgNPs} / \mathrm{TXICh}$ prepared in the presence of triethanolamine.

For AgNPs/TXICh/TEOH, the micrographs (Figures 4c and $4 \mathrm{~d}$ ) revealed stable nanoparticles with a spherical shape and a relative narrow particle distribution around $2.5 \mathrm{~nm}$ (inset of Figure 4d). This experimental observation can be explained by the initiation mechanism. The particle formation by the reduction of $\mathrm{Ag}^{+}$by the TXICh radical is relatively slow, since the electrochemical potential of the radical is probably more positive than that of the $\mathrm{Ag}^{+} / \mathrm{Ag}^{\mathrm{o}} .{ }^{44,45}$ However, the reaction is fast enough to compete with the mutual deactivation of the radical at 

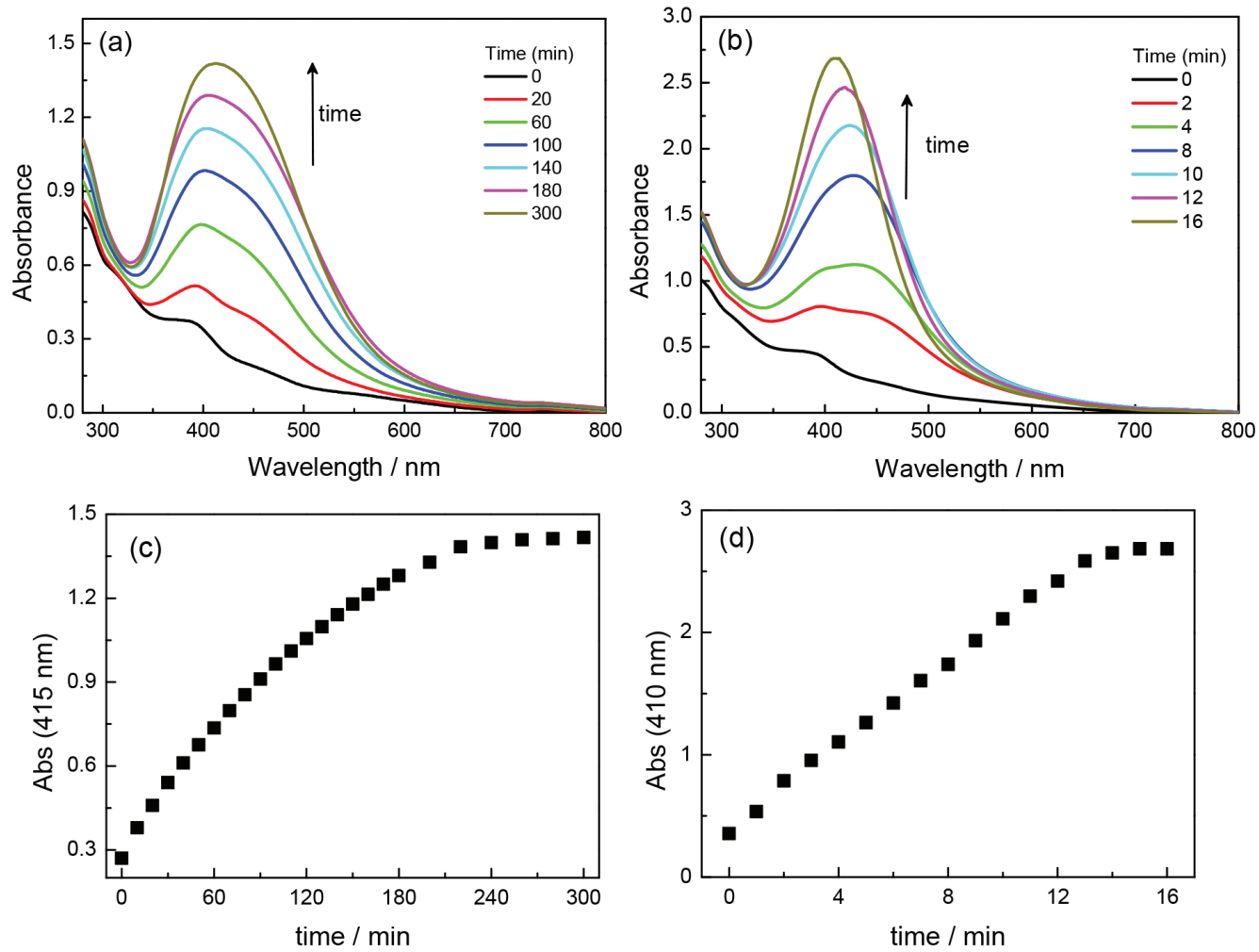

Figure 3. Absorption spectra of AgNP synthesized by using TXICh in the absence (a) and presence (b) of TEOH as a function of irradiation time. And (c) and (d), time evolution of the absorbance at the maximum for the systems above.
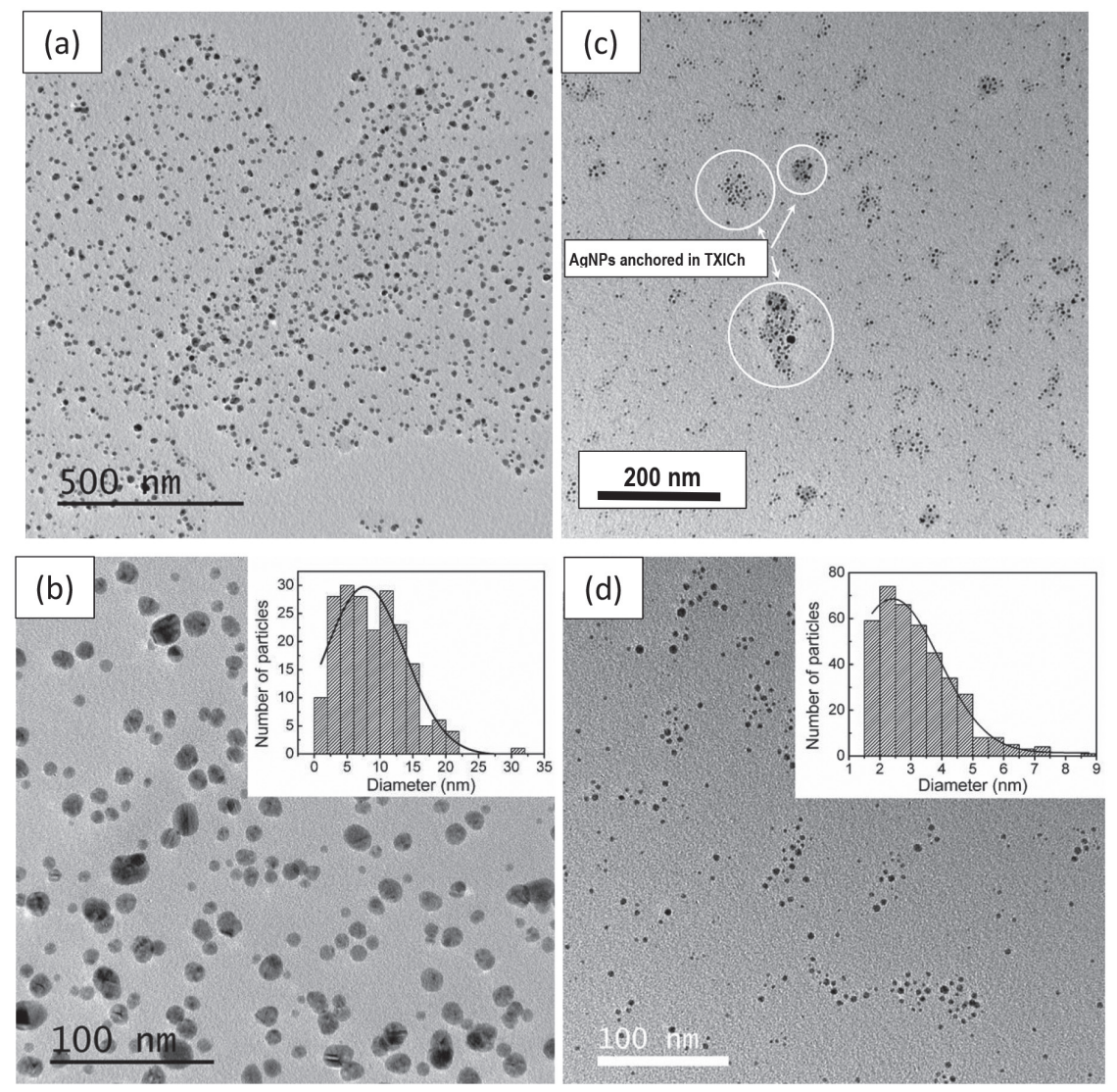

Figure 4. Transmission electron micrographs of AgNPs/TXICh (a) and (b), TEM images of AgNPs/TXICh in the presence of TEOH (c) and (d). Inserts: particle size distribution estimated from TEM images. 
the used light intensity. On the other hand, the reaction involving ethanolamine is much faster, as evidenced by the maximum conversion of $\mathrm{Ag}^{+}$ions into nanoparticles reached after about $15 \mathrm{~min}$ (see Figure 3d).

In both systems, in the presence and absence of TEOH, the silver nanoparticles are stabilized by the TXICh modified chitosan. The TEM images (Figure 4c) suggest that the AgNPs were mainly anchored on the TXICh particles.

\section{Synthesis and characterization of AuNP/TXICh}

Gold nanoparticles were photochemically synthesized by irradiation of a solution of $\mathrm{AuCl}_{4}{ }^{-}$ions in the presence of TXICh acting simultaneously as photoreducing agent and stabilizer. The mixtures were irradiated using a LED at $405 \mathrm{~nm}$.

During irradiation the plasmon band at $530 \mathrm{~nm}$ started growing and after about $6 \mathrm{~min}$ the solution became reddish and the absorption spectra remained stable (Figure 5a), indicating that at that point the conversion of $\mathrm{Au}^{+}$to $\mathrm{AuNP}$
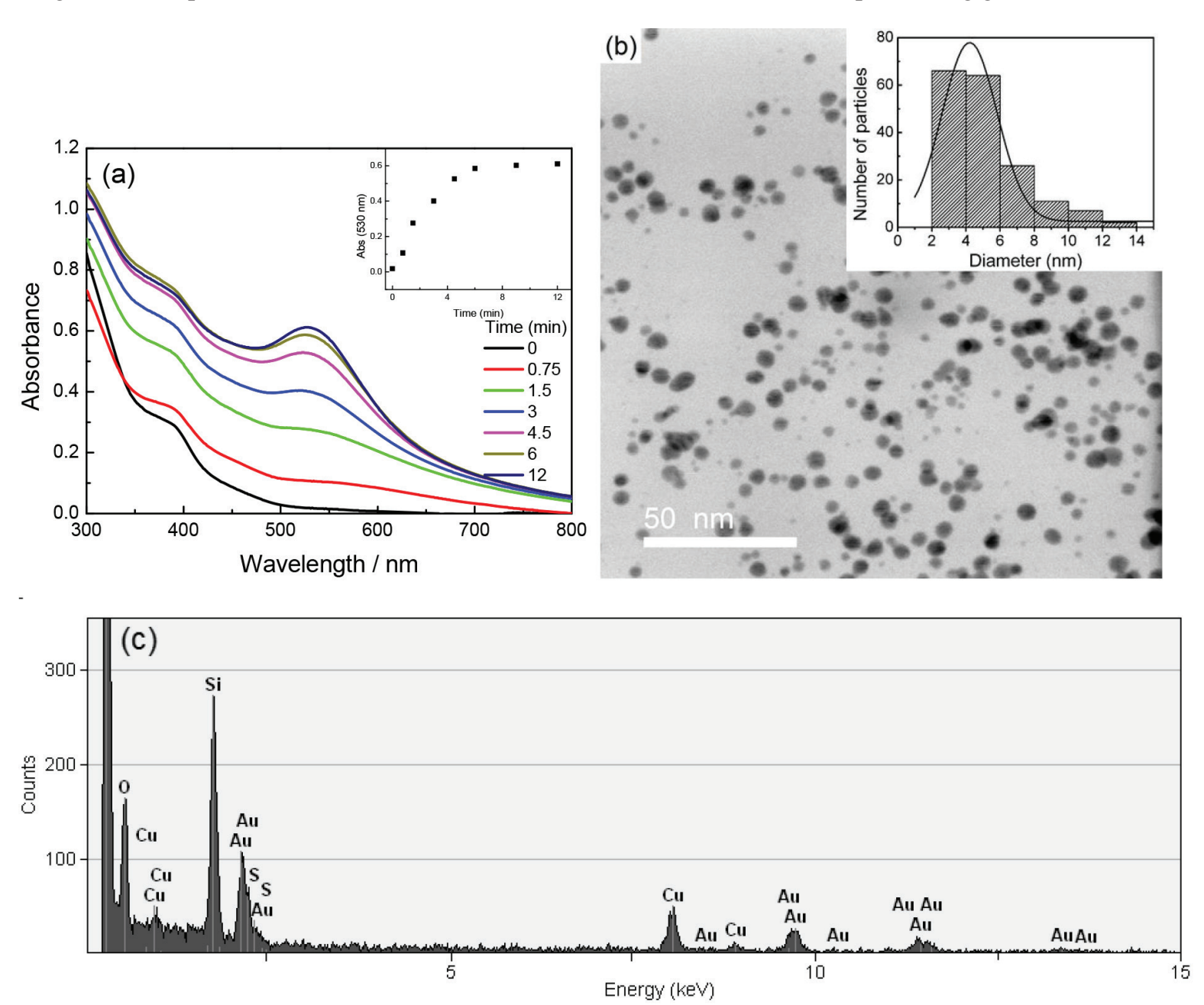

Figure 5. (a) Absorption spectra as a function of irradiation time, (b) transmission electron micrographs (inset: particle size distribution estimated from

TEM images), and (c) EDS spectrum of AuNP/TXICh. was completed. The UV-Vis spectra of AuNPs prepared using $405 \mathrm{~nm}$ LED as light source is shown in Figure 5a. ${ }^{46}$

It is worth noting that the bands assigned to the TXICh (around $380 \mathrm{~nm}$ ) remain unchanged during the irradiation. This behavior can be attributed to the regeneration of the thioxanthones, which occurs simultaneously with the reduction of the metallic ions. According to Malval et al., ${ }^{42}$ thioxanthone and derivatives regeneration may occur due to the oxidation of the ketyl anion by the metallic ions.

Gold nanoparticles/TXICh were further characterized by TEM (Figure $5 b$ ). TEM images revealed that the formed AuNPs/TXICh are spherical with a narrow size distribution with average diameter of about $4 \mathrm{~nm}$. Energy dispersive $\mathrm{X}$-ray analysis (EDX) was used to examine the presence of elemental gold in the formed NPs. Figure 5c shows EDS spectrum of AuNPs/TXICh. The signal around $2 \mathrm{keV}$ confirms the presence of elemental gold in the solution. In addition, the signals corresponding to carbon, oxygen and sulfur of the TXICh species, as well as the presence of atoms from the sample holding grid are detected. 


\section{Conclusions}

The ability of 10-oxo- $10 H$-dibenzene thiopyran3-4-dicarboximide chitosan (TXICh) to act as reducing agent and stabilizer in the synthesis of silver and gold nanoparticles was presented in this work. Silver nanoparticles were prepared using TXICh in the absence and presence of triethanolamine (TEOH). For both systems, the AgNPs/TXICh nanoparticles were observed by the appearance of the plasmon absorption in the range of 410-415 $\mathrm{nm}$ and electron microscopy images revealed AgNPs with spherical shape. The synthesis of AgNPs using TXICh in the absence of TEOH occurred slowly (around $4 \mathrm{~h}$ ), whereas for AgNPs prepared in the presence of TEOH maximum conversion was obtained after $15 \mathrm{~min}$. Moreover, the AgNPs nanoparticles prepared with $\mathrm{TXICh} / \mathrm{TEOH}$ were smaller and showed a more uniform size. For AuNPs prepared using TXICh as photoinitiator system, the nanoparticles showed an intense surface plasmon band at $530 \mathrm{~nm}$ and the TEM images revealed particles with spherical profiles and particle size around $4 \mathrm{~nm}$. In summary, uniform and small size AgNPs and AuNPs can be prepared by a photochemical procedure using TXICh simultaneously as photoinitiator and stabilizer.

\section{Supplementary Information}

Supplementary data are available free of charge at http://jbcs.sbq.org.br as PDF file.

\section{Acknowledgments}

This work was supported by CNPq (490421/2013-0, 304579/2016-5), FAPESP (17/16914-2, 12/19656-0). C. C. S. and M. G. N. thank CNPq for research fellowships. S. R. V. thanks CAPES for a graduate fellowship.

\section{References}

1. Dubas, S. T.; Kumlangdudsana, P.; Potiyaraj, P.; Colloids Surf., A 2006, 289, 105.

2. Lombardo, P. C.; Poli, A. L.; Castro, L. F.; Perussi, J. R.; Schmitt, C. C.; ACS Appl. Mater. Interfaces 2016, 8, 21640.

3. Zhang, J. Z.; Noguez, C.; Plasmonics 2008, 3, 127.

4. Oh, H. S.; Liu, S.; Jee, H. ; Baev, A.; Swihart, M. T.; Prasad, P. N.; J. Am. Chem. Soc. 2010, 132, 17346.

5. Henglein, A.; Chem. Rev. 1989, 89, 1861.

6. Talapin, D. V.; Lee, J. S.; Kovalenko, M. V.; Shevchenko, E. V.; Chem. Rev. 2010, 110, 389.

7. Stratakis, M.; Garcia, H.; Chem. Rev. 2012, 112, 4469.

8. Takai, A.; Kamat, P. V.; ACS Nano 2011, 5, 7369.
9. Sonavane, G.; Tomoda, K.; Sano, A.; Ohshima, H.; Terada, H.; Makino, K.; Colloids Surf., B 2008, 65, 1.

10. Corbierre, M. K.; Cameron, N. S.; Lennox, R. B.; Langmuir 2004, 20, 2867.

11. Scaiano, J. C.; Billone, P.; Gonzalez, C. M.; Maretti, L.; Marin, M. L.; McGilvray, K. L; Yuan, N.; Pure Appl. Chem. 2009, 81, 635.

12. Lee, J.; Lee, M.; J. Phys. Chem. C 2016, 120, 13256.

13. Amendola, V.; Meneghetti, M.; Phys. Chem. Chem. Phys. 2009, 11, 3805 .

14. Binaymotlagh, R.; Hadadzadeh, H.; Farrokhpour, H.; Haghighi, F. H.; Abyar, F.; Mirahmadi-Zare, S. Z.; Mater. Chem. Phys. 2016, 177,360 .

15. Sakamoto, M.; Fujistuka, M.; Majima, T.; J. Photochem. Photobiol., C. 2009, 10, 33.

16. Eguchi, M.; Ito, M.; Ishibashi, T.; Chem. Lett. 2014, 43, 140.

17. Contin, A.; Biffis, A.; Sterchele, S.; Dormbach, K.; Schipmann, S.; Pich, A.; J. Colloid Interface Sci. 2014, 414, 41.

18. Mallick, K.; Wang, Z. L.; Pal, T.; J. Photochem. Photobiol., A 2001, 140, 75.

19. Wojtysiak, S.; Kudelski, A.; Colloids Surf., A 2012, 410, 45.

20. Chhatre, A.; Solasa, P.; Sakle, S.; Thaokar, R.; Mehra, A.; Colloids Surf., A 2012, 404, 83.

21. Dey, S.; Sherly, M. C. D.; Rekha, M. R.; Sreenivasan, K.; Carbohydr. Polym. 2016, 136, 71.

22. Seo, H. J.; Kim, J. C.; Colloid Polym. Sci. 2015, 293, 1425.

23. Dutta, P. K.; Dutta, J.; Tripathi, V. S.; J. Sci. Ind. Res. 2004, 63, 20.

24. Kumar, M. N. V. R.; React. Funct. Polym. 2000, 46, 1.

25. Pillai, C. K. S.; Paul, W.; Sharma, C. P.; Prog. Polym. Sci. 2009, 34, 641 .

26. Rinaudo, M.; Prog. Polym. Sci. 2006, 31, 603.

27. Long, D. W.; Wu, G. Z.; Chen, S. M.; Radiat. Phys. Chem. 2007, 76, 1126.

28. Du, Y.; Luo, X. L.; Xu, J. J.; Chen, H. Y.; Bioelectrochemistry 2007, 70, 342.

29. Fan, C. L.; Li, W.; Zhao, S. J.; Chen, J.; Li, X.; Mater. Lett. 2008, 62, 3518.

30. Huang, H. Z.; Yang, X. R.; Biomacromolecules 2004, 5, 2340.

31. Huang, L.; Zhai, M. L.; Peng, J.; Xu, L.; Li, J. Q.; Wei, G. S.; J. Colloid Interface Sci. 2007, 316, 398.

32. Wei, D. W.; Qian, W. P.; Colloids Surf., B 2008, 62, 136.

33. Cheng, D. M.; Zhou, X. D.; Xia, H. B.; Chan, H. S. O.; Chem. Mater. 2005, 17, 3578.

34. Valandro, S. R.; Poli, A. L.; Venancio, T.; Pina, J.; de Melo, J. S.; Burrows, H. D.; Schmitt, C. C.; J. Photochem. Photobiol., A 2016, 327, 15.

35. Fecchio, B. D.; Valandro, S. R.; Neumann, M. G.; Cavalheiro, C. C. S.; J. Braz. Chem. Soc. 2016, 27, 278.

36. Valandro, S. R.; Poli, A. L.; Neumann, M. G.; Schmitt, C. C.; Appl. Clay Sci. 2013, 85, 19. 
37. Balta, D. K.; Arsu, N.; Yagci, Y.; Jockusch, S.; Turro, N. J.; Macromolecules 2007, 40, 4138.

38. Corrales, T.; Catalina, F.; Peinado, C.; Allen, N. S.; Rufs, A. M.; Bueno, C.; Encinas, M. V.; Polymer 2002, 43, 4591.

39. Karaca, N.; Ocal, N.; Arsu, N.; Jockusch, S.; J. Photochem. Photobiol., A 2016, 331, 22.

40. Kork, S.; Yilmaz, G.; Yagci, Y.; Macromol. Rapid Commun. 2015, 36, 923.

41. Ferreira, G. C.; Schmitt, C. C.; Neumann, M. G.; J. Braz. Chem. Soc. 2006, 17, 905 .
42. Malval, J. P.; Jin, M.; Balan, L.; Schneider, R.; Versace, D. L.; Chaumeil, H.; Defoin, A.; Soppera, O.; J. Phys. Chem. C 2010, $114,10396$.

43. Liu, B.; Li, X. Y.; Zheng, C. F.; Wang, X. Y.; Sun, R. C.; Nanotechnology 2013, 24.

44. Henglein, A.; Giersig, M.; J. Phys. Chem. B 1999, 103, 9533.

45. Henglein, A.; Chem. Mater. 1998, 10, 444.

46. Orendorff, C. J.; Sau, T. K.; Murphy, C. J.; Small 2006, $2,636$.

Submitted: January 29, 2019

Published online: August 6, 2019 